Gran dependencia

\section{y uso de servicios sanitarios: ¿un exceso de demanda?}

\section{Severe level of dependence and use of health services: Excessive demand?}

José Manuel Jiménez Rodríguez

Carmen Fajardo Contreras ${ }^{2}$

Mohamed Farouk Allam ${ }^{3}$

1. Antropólogo Social//rabajador Social. Unidad de Gestión Clínica Rute/lznajar. Distrito Sanitario Córdoba Sur.

2. Médico de Familia. Unidad de Gestión Clínica Rute. Distrito Sanitario Córdoba Sur.

3. Responsable de Epidemiología e Investigación. Distrito Sanitario Córdoba Sur, Lucena.

\section{Correspondencia:}

Mohamed Farouk Allam

Departamento de Ciencias Sociosanitarias y Radiología y Medicina Física.

Facultad de Medicina, Universidad de Córdoba

Avda. Menéndez Pidal, s/n

14004 Córdoba

Tel. + (34) 957218278

$\mathrm{Fax}+(34) 957218573$

E-mail: fm2faahm@uco.es

\section{RESUMEN}

Objetivos: el objetivo de nuestro estudio es conocer el grado de uso de los servicios sanitarios de las personas mayores y/o discapacitadas en situación de gran dependencia diagnosticada.

Diseńo: estudio descriptivo transversal.

Emplazamiento: municipio de Rute. Provincia de Córdoba (España). Población y muestra: el universo muestral de pacientes en situación de gran dependencia residente en el municipio de Rute (Córdoba, España), desde el 1 de enero de 2010 al 31 de diciembre de 2010.

Resultados: se incluyeron 73 personas. La media de edad fue de 75,2 (Dt 19,3; rango 12-98) y el 61,6\% fueron mujeres. La media de consultas en centro/domicilio médicas, de enfermería, de gestoras de casos y de trabajadores sociales fueron 22,4 (Dt 24,4)/0,52 (Dt 1,6), 8,6 (Dt 6,7)/7,9 (Dt 21,9), 0,88 (Dt 1,4)/1 (Dt 1,2) y 0,60 (Dt 1,2)/0,1 (Dt 0,30), respectivamente. La media de urgencias en atención primaria fue de 4,97 (Dt 10,9) y la de hospitalarias 1,05 (Dt 1,8$)$, con una media de 1,99 (Dt 3,7) derivaciones programadas a especialidades.

Conclusión: La gran dependencia ocasiona un consumo elevado de consultas médicas y de enfermería, por lo que es un factor clave en la planificación de recursos sanitarios.

PALABRAS CLAVE: gran dependiente, gasto sanitario, Rute, envejecimiento.

\section{ABSTRACT}

Objectives: The objective of our study is to evaluate the magnitude of use of health services by elderly and/or disabled patients with severe level of dependence.

Design: Transversal descriptive study.

Location: Village of Rute. Province of Cordoba (Spain).

Study population and sample: All elderly and/or disabled patients with severe level of dependence living in the village of Rute (Cordoba, Spain), from January 1, 2010 to December 31, 2010.

Results: 73 elderly and/or disabled patients with severe level of dependence were included. The average age was of 75.2 (SD19.3, range $12-98)$ and $61.6 \%$ were women. The mean number of Primary Healthcare Centre(PHC)/Home Visits of family doctor, nurse, social worker and case management nurse were 22.4 (SD 24.4)/0.52 (SD 1.6), 8.6 (SD 6.7)/7.9 (SD 21.9), 0.88 (SD 1.4)/1 (SD 1,2) and 0.60 (SD 1.2)/0.1 (SD 0.30) respectively. The average PHC emergency visits was 4.97 (SD 10.9) and hospital emergency visits 1.05(SD 1.8), with an average of 1.99 (SD 3.7) scheduled specialty referrals. Conclusion: Elderly and/or disabled patients with severe level of dependence demand important number of medical and nursing visits. Needs of elderly and/or disabled patients should be considered in all public health policies and plans.

KEYWORDS: Severe level of dependence, health costs, Rute, aging.

\section{- INTRODUCCIÓN}

A lo largo de las últimas décadas, España, al igual que otros países de la Unión Europea (UE), ha sufrido cambios importantes en términos sociodemográficos, cambios que han afectado directa e indirectamente a la política social y sanitaria, así como a su forma de entender las necesidades sociales y prestaciones asistenciales, entre otras.

Parte de estos cambios sientan su base en nuestro estado de bienestar o, como apuntan algunos autores, el estado social o socialdemócrata, siendo este pilar fundamental y eje central en el que nos apoyamos a la hora de hablar de políticas de igualdad y derechos sociales, como son, entre otros, el derecho a una sanidad pública y gratuita (art. 50 de la Constitución Española $[\mathrm{CE}])^{1}$. De igual modo, el estado del bienestar al que nos referimos hace patente la necesidad de promover y mantener una red asistencial de apoyo social y sanitario que actúe como colchón protector y contribuya a la sostenibilidad socioeconómica y sanitaria de la comunidad, evitando con ello el favorecimiento de la pauperización ${ }^{2,3}$.

Los avances técnicos y tecnológicos, los adelantos de la medicina y la investigación, la farmacología y los nuevos estilos de vida han ocasionado un mayor bienestar y una mejor calidad de vida en las personas, provocando con ello una mayor longevidad y un envejecimiento sin precedente. En otro sentido, esto ha dado origen a la aparición de nue- 
vas enfermedades y/o patologías no investigadas o desconocidas para la medicina y el campo de la salud ${ }^{4}$; sea como fuere, dicha tendencia lleva aparejada una mayor visualización de personas con edad superior a los 65 ańos, por un lado, así como el descenso de la natalidad en los hogares y las familias extensas, por otro. Como consecuencia de todos estos factores (medicina, farmacología y sistema de salud actual) influyentes en los procesos asistenciales de salud y enfermedad de las personas, la pirámide de población ha experimentado un notable cambio y ha quedado de manera invertida. Todo ello tiene como resultado la actual situación de dependencia por la que muchos individuos y familias se ven afectados: pacientes con enfermedades crónicas y/o degenerativas y que producen en quien las padece situaciones y/o estados de déficit y descompensación biopsicosocial.

Dicha situación ha originado en el engranaje del estado del bienestar la vertebración de un cuarto pilar sobre el que descansa la dependencia y que va a servir de guía a las políticas redistributivas e igualitarias ${ }^{5}$; dependencia que, y en un intento de solventar desajustes socioeconómicos y familiares, pone en marcha un sistema de protección social basado en la ayuda al enfermo y la familia, mediante aportaciones económicas o asistencia social domiciliaria, para personas enfermas y discapacitadas.

Las limitaciones funcionales, la dificultad para el desarrollo de las actividades de la vida diaria, la falta de recursos económicos y/o humanos hacen que muchas de estas personas dependientes vivan su situación de un modo estresante y carente de los medios necesarios para el mejor desarrollo, integración e inclusión social. Asimismo, dicha situación perjudica sobremanera el sistema de protección social (pensiones y prestaciones, sanidad y servicios sociales), motivo por el cual los poderes públicos se han visto obligados a paliar y minimizar las carencias con las que cuenta el sistema ${ }^{7}$. Es en el año 2007 cuando España, igual que otros países de la UE, implanta como medida correctora la ley 39/2006, de 14 de diciembre, de Promoción de la Autonomía Personal y Atención a las personas en situación de dependencia, o como comúnmente se la conoce "ley de la dependencia". Con ella se pretende regularizar desajustes que han venido produciéndose a lo largo de años y que afectaban tanto al que la padece como a los cuidadores y la propia administración pública, esta última obligada a dar el tratamiento necesario que dicha cuestión suscita en el régimen del bienestar tanto a nivel español como europeo?.

Desde que esta ley se ha puesto en marcha, quedando recogido en el articulado su catálogo de prestaciones, el consumo de recursos sociales y sanitarios se ha visto incrementado y desbordado. Atendiendo al sistema sanitario español y por defecto al andaluz, por ser el que nos toca de lleno, el número de avisos y visitas domiciliarias, de consultas médicas, de enfermería y de trabajo social, el número de informes médicos y enfermería, así como las derivaciones han incrementado su porcentaje. Ahora son más las personas dependientes las que quieren beneficiarse de las ventajas que esta lleva aparejadas, y se han convertido en grandes consumidores de recursos sociosanitarios y de tiempo libre del cuidador $^{10}$. Por su parte, los cuidadores informales pasan a formar parte de los activos de salud y agentes de cambio de estos dependientes, actuando en aras de la contención de situaciones de necesidad y dependencia de larga duración, con las repercusiones que los cuidados y la sobrecarga familiar puedan tener sobre las capacidades físicas y psicológicas de los que cuidan ${ }^{11}$.

El objetivo de este estudio es conocer el grado de uso de los servicios sanitarios públicos de las personas mayores y/o discapacitadas en situación de gran dependencia diagnosticada. Los recursos públicos sanitarios se están viendo perjudicados, entre otros motivos, por la hiperfrecuentación, y aun siendo estos un recurso universal, pueden verse colapsados y generar desigualdades sociales y sanitarias ${ }^{12}$.

\section{- METODOLOGÍA}

La metodología llevada a cabo en nuestra investigación está basada fundamentalmente en los estudios de cohorte cuantitativa. Para ello hemos tomado como referencia el modelo biopsicosocial basándonos en un paradigma positivista.

Se trata de un estudio descriptivo trasversal mediante el cual pretendemos conocer el estado actual de la situación de gran dependencia (Grado III, Nivel 1 y 2) para poder establecer un perfil concreto y ajustado con el que cuentan los individuos que la padecen en la zona básica de salud (ZBS) de Rute (Córdoba, Espańa), así como aproximarnos a los costes indirectos derivados de esta en la administración pública y en concreto el sistema andaluz de salud. Se tendrán en cuenta para ello indicadores de tipo cuantitativo que nos aportarán cifras objetivables sobre la situación real de la dependencia dentro del propio Distrito Sanitario Córdoba Sur, a fin de poder establecer comparaciones con otra ZBS del mismo distrito sanitario, para lo cual se ha realizado un análisis profundo y exhaustivo de la situación de gran dependencia.

Las técnicas e instrumentos utilizados en dicha investigación están basados en el análisis primario de datos, en donde, para la recogida, análisis e interpretación de los datos obtenidos hemos empleado como herramientas de trabajo los siguientes instrumentos:

\section{PARA LA RECOGIDA Y MANIPULACIÓN DE DATOS}

- Programa informático Excel.

- Programa informatizado del SAS (Diraya: Historia Única).

- BDU (Base de Datos de Usuario).

- Programa SIUSS (Sistema Informático de Usuarios de Servicios Sociales) del Instituto Provincial de Bienestar Social (IPBS) de la Diputación de Córdoba.

- Programa Informático del INSS.

\section{Cronograma}

Dicho estudio se realiza respetando la cronología y plazos que a continuación se reflejan: en enero de 2010, se hace la selección de la muestra. El mes de febrero del mismo ańo está dedicado a la búsqueda de información, remitiéndonos a las fuentes previamente establecidas. El plazo que va de marzo a junio se dedica a la recogida de datos, y el mes de julio a su análisis. Finalmente y tras un mes de descanso, destinamos el mes de septiembre a establecer e interpretar los resultados que se muestran en la tabla 1.

Una vez elaborado el diseño de investigación y las líneas estratégicas, así como definido el número real de usuarios/pacientes grandes dependientes incluidos en el estudio, se ha dado paso a la recogida de datos en formato "Excel". Para ello hemos contado con el consentimiento y aprobación del Distrito Sanitario Córdoba Sur. Asimismo, hemos contado con el apoyo del personal facultativo, la enfermería gestora de casos y la unidad de trabajador social, quienes se han prestado a colaborar a través de la facilitación de datos y el recuento de dependientes entre el 1 enero de 2010 y el 31 de diciembre de 2010. Del mismo modo, nos han facilitados las claves de acceso al programa informático de salud Diraya y BDU.

De esta manera, se puede decir que para la recogida de datos no ha sido necesaria ni la participación ni el contacto directo con los usuarios/pacientes, como tampoco se ha necesitado de su aprobación o consentimiento, por mantener la privacidad y el anonimato de estos, siguiendo para ello los preceptos que marcan la ley de protección 
Tabla 1. Establecimiento e interpretación de los resultados

\begin{tabular}{|l|c|c|c|c|c|c|c|c|}
\hline & Enero & Febr. & Marzo & Abril & Mayo & Junio & Julio & Agosto \\
\hline Selección de la muestra & $X$ & & & & \\
\hline Búsqueda de información & & $X$ & & & & \\
\hline Recogida de datos & & & $X$ & $X$ & $X$ & $X$ & $X$ \\
\hline Análisis de los datos & & & & & \\
\hline Interpretación/resultados & & & & \\
\hline
\end{tabular}

de datos de carácter personal, la ley de autonomía del paciente y el código deontológico.

\section{Para el análisis de Datos:}

Los datos recogidos fueron analizados estadísticamente mediante el programa EPIDAT versión 3.1.

\section{- RESULTADOS}

Del estudio realizado en el municipio de Rute (Córdoba, España), donde el número total de habitantes para el año 2010 asciende a un total de 10000 aproximadamente, y donde las personas en situación de gran dependencia representan menos del $5 \%$, se desprenden los siguientes resultados: del total de 78 personas, 66 son mayores de 65 años; la media de edad es de $75+20$ años (rango 12-98) y el 60,3\% son mujeres. La utilización de recursos públicos a nivel sanitario en atención primaria, así como en urgencias y el ámbito hospitalario, se resumen en las tablas 2 y 3 .

\section{- DISCUSIÓN}

Es cada vez más fácil encontrarnos con sociedades modernas donde el perfil poblacional lo constituyen mayoritariamente personas longevas. Se trata de sociedades envejecidas (clases pasivas), consumidoras de prestaciones y recursos sociales, donde las aportaciones y contribuciones al sistema sanitario y de seguridad social son escasas, por no decir nulas. Ello está generando un colapso sanitario y también que otras administraciones públicas se vean abordadas por una situación donde las necesidades sociosanitarias superan a las expectativas de la política social y de igualdad, así como a sus presupuestos generales.

Con este trabajo nos planteamos hacer un análisis pormenorizado sobre el uso de los servicios sanitarios generado por la gran dependencia en el municipio de Rute, así como hacer reflexionar sobre qué medidas deberían ser las más adecuadas para paliar dicha situación. De acuerdo con ello, así como con los resultados obtenidos de dicha investigación, podemos afirmar que son mayoritariamente los facultativos y los enfermeros comunitarios (AP) quienes a través de la atención directa y la visita domiciliaria soportan el peso de la gestión de este colectivo, mayoritariamente femenino y mayor de 65 años; estos realizan una labor de contención y mantenimiento del paciente en su domicilio u entorno habitual, evitando ingresos hospitalarios innecesarios, traducido en un reducido número de derivaciones hospitalarias programadas, a la mitad, si se compara con las urgencias en AP. De igual modo, si tenemos en cuenta el volumen de consultas y avisos domiciliarios por parte de los facultativos con respecto a este tipo de pacientes, las derivaciones y urgencias cargadas al hospital no superan el 3,3 de media.
Tabla 2. Utilización de recursos públicos sanitarios por grandes dependientes en atención primaria

\begin{tabular}{|c|c|c|c|}
\hline Consulta de atención primaria (AP) & Media & $\begin{array}{l}\text { Desviación } \\
\text { típica }\end{array}$ & Rango \\
\hline Consulta médica de AP & 22 & 14,6 & $0-61$ \\
\hline Visita domiciliaria del médico de AP & 0,6 & 1,7 & $0-9$ \\
\hline Consulta de enfermería de AP & 8,3 & 6,7 & $0-27$ \\
\hline Visita domiciliaria de enfermería de AP & 8,5 & 21,9 & $0-159$ \\
\hline Consulta de la enfermera gestora de casos & 0,9 & 1,4 & $0-9$ \\
\hline $\begin{array}{l}\text { Visita domiciliaria de la enfermera gestora } \\
\text { de casos }\end{array}$ & 1 & 1,2 & $0-6$ \\
\hline Consulta del trabajador social de AP & 0,6 & 1,2 & $0-7$ \\
\hline Visita domiciliaria del trabajador social de AP & 0,9 & 0,3 & $0-2$ \\
\hline
\end{tabular}

Tabla 3. Utilización de recursos públicos sanitarios por grandes dependientes en urgencias, atención primaria y atención hospitalaria

\begin{tabular}{|l|c|c|c|}
\hline & Media & $\begin{array}{c}\text { Desviación } \\
\text { típica }\end{array}$ & Rango \\
\hline Urgencias de atención primaria & 4,7 & 10,6 & $0-70$ \\
\hline $\begin{array}{l}\text { Urgencias del Hospital Infanta Margarita, } \\
\text { Cabra }\end{array}$ & 1,2 & 2,1 & $0-11$ \\
\hline Derivaciones hospitalarias programadas & 2,1 & 3,4 & $0-25$ \\
\hline
\end{tabular}

Ello hace que sea el equipo interdisciplinar de AP quien deba dar solución con los recursos existentes a las exigencias demandadas por los grandes dependientes y sus familiares y/o cuidadores.

La situación de dependencia en general y de gran dependencia en particular lleva aparejado un déficit socioeconómico importante, en tanto en cuanto se trata de personas que están a merced de una tercera para todas las AVD y requieren de la protección social de la administración pública la cual, a través del diseño de estrategias y políticas públicas debe dar respuesta a las necesidades planteadas por estas personas.

En conclusión, la gran dependencia ocasiona un consumo elevado de consultas médicas y de enfermería, por lo que es un factor clave en la planificación de recursos sanitarios, pues con ello se evita el colapso sanitario y se garantiza la sostenibilidad del sistema público de salud, sin que se vean dańados los intereses de los principales interesados, los pacientes 


\section{- BIBLIOGRAFÍA}

1. Constitución Española. Secretaria General del Senado. 5. ${ }^{\text {a ed }}$ Madrid: Biblioteca Nueva; 2010

2. Cohen MA, Méndez LH. La Sociedad del Riesgo: Amenaza y Promesa. Sociológica. 2000;15(43):173-201.

3. Sotelo I. El Estado Social: Antecedentes, origen, desarrollo y declive. Fundación Alfonso Martín Escudero. Madrid: Editorial Trotta; 2010.

4. Bertrand G, Hiernaux D, Lindon A. Tratado de geografía humana. Barcelona: Anthropos: 2006

5. Beltrán M. Burguesía y Liberalismo en la España del siglo XXI. Granada: Edita Universidad de Granada; 2010.

6. III Plan Andaluz de Salud 2003-2008. Convergencias. Plan de apoyo a la familia. Línea prioritaria de actuación 4 y siguiente. Sevilla: Junta de Andalucía; 2003. p. 83-109.

7. Garcés J, Ródenas F, Sanjosé V. Towards a new welfare state: the social sustainability principle and health care strategies. Health Policy. 2003;65:201-15

8. Ley de Promoción de la Autonomía Personal y Atención a las personas en situación de Dependencia. L. No. 39 (Dic. 14, 2006).

9. Arriba A, Moreno FJ. El tratamiento de la dependencia en los regímenes de bienestar europeo contemporáneos. Colección estudios. Serie dependencia. Secretaría general de política social y consumo. Madrid: IMSERSO; 2009.
10. Durán MA. Los Costes Invisibles de la Enfermedad. 2. ed. Madrid: Fundación BBVA; 2003

11. Zarit S, Leitsch S. Applications of personal control. En: Zarit SH, Pearlin LI, Schaie KW, editores. Personal control in so cial and life course contexts. New York: Springer; 2002; p. 281-300

12. OMS. Comisión sobre determinantes sociales en salud. Subsanar las desigualdades en una generación. Alcanzar la equidad sanitaria actuando sobre los determinantes sociales en salud. Disponible en: http://www.who.int/social_determinants/final_report/csdh_finalreport_2008_execsumm_es.pd 